\title{
A Case of Primary Myxoid Leiomyosarcoma of the Liver
}

\author{
Heon Ju Lee, M.D., Jae Lyun Lee, M.D. and Won Hee Choi, M.D.*
}

Departments of Internal Medicine and Pathology*,

Yeungnam University College of Medicine, Taegu, Korea

\begin{abstract}
Primary hepatic leiomyosarcoma is very rare and no primary myxoid leiomyosarcoma in the liver has been reported yet. Most cystic space-occupying lesions in the liver are benign in nature. But, rarely, malignancy could appear as a cystic lesion by ultrasonographic examination. A 64-year-old woman with a huge cystic mass detected by hepatic ultrasonography was diagnosed as primary hepatic myxoid leiomyosarcoma by immunohistochemical and ultrastructural studies after various image studies and fine needle aspiration biopsy of the liver mass.
\end{abstract}

Key Words : Leiomyosarcoma, Liver neoplasm

\section{INTRODUCTION}

More than 25 cases of primary hepatic leiomyosarcoma have been reported from many countries since the first publication in Japan in 1995 ${ }^{1-4)}$. Myxoid leiomyosarcoma is a distinctive and rare tumor found in many organs, such as the digestive tracts, uterus, ovary and prostate $^{5-7)}$, but has not previously been reported in the liver.

In Korea, primary malignant hepatic neoplasm is the third leading cause of death ${ }^{8)}$ and the most common hepatic neoplasm is hepatocellular carcinoma related to chronic hepatitis $\mathrm{B}^{9}$. Another common space-occupying lesion in the liver is the benign $\mathrm{cyst}^{10)}$. Therefore, differential diagnosis of hepatic masses is very important.

We report a case of primary hepatic myxoid leiomyosarcoma which was treated successfully by extended right lobectomy and appears to be the first case in English literature.

Address reprint requests to : Heon Ju Lee, M.D., Department of Internal Medicine, College of Medicine, Yeungnam University, Dae-Myung Dong, Nam Gu, Daegu, 705-035, Korea

\section{CASE REPORT}

A 64-year-old Korean woman visited the out-patient clinic of the Department of Internal Medicine of Yeungnam University Medical Center complaining of a palpable mass with dull pain on the right upper quadrant for 10 days. She was referred from a local clinic with the report of a huge cystic mass detected by ultrasonography. The pain was not like biliary colic in nature and she had no other symptom. The patient was a housewife, non-alcohol drinker and non-smoker. She had never been abroad. She had a medical history of a subtotal gastrectomy with gastrojejunostomy 7 years earlier for the treatment of benign gastric ulcer bleeding.

The palpable portion of the liver was non-tender and no bruit was heard over it. Splenomegaly, spider angioma, superficial venous collaterals and jaundice were not detected.

Liver function test results were abnormal; AST $48 \mathrm{IU} / \mathrm{L}$ (normal 13 35 $\mathrm{IU} / \mathrm{L}$ ), ALT $26 \mathrm{IU} / \mathrm{L}$ (normal 9 40 $\mathrm{IU} / \mathrm{L}$ ), ALP $242 \mathrm{IU} / \mathrm{L}$ (normal 26 88 IU/L), v-GTP $165 \mathrm{IU} / \mathrm{L}$ (normal 8 64 IU/L), and LDH $620 \mathrm{IU} / \mathrm{L}$ (normal 150 550 $\mathrm{IU} / \mathrm{L}$ ). Tests for hepatitis $\mathrm{B}$ and hepatitis $\mathrm{C}$ were all negative, and serum a-fetoprotein was within the normal limit. All other laboratory findings were normal.

Chest radiography was normal, but a large soft-tissue 
mass was apparent in the right upper quadrant on a plain abdominal film. Ultrasonography of the hepatobiliary system showed a mixed cystic lesion measuring $14.5 \mathrm{~cm}$ in diameter and occupying almost all of the right lobe of the liver. Contrast enhanced abdominal computed tomographic (CT) scan showed a thick-walled cystic mass of $15 \mathrm{~cm}$ in diameter in the right hepatic lobe with serpentine enhancement in the wall during the early arterial contrast phase (Figure 1). Pelvic CT showed normal uterus and adnexa. Angiography showed a hypervascular tumor which displaced hepatic artery branches in segments 5, 6, 7 and 8, and neovascularization was noted at the area of segments 4, 5, 6, 7, and 8 (Figure 2). Technetium ${ }^{99 m}$ phytate liver scan showed

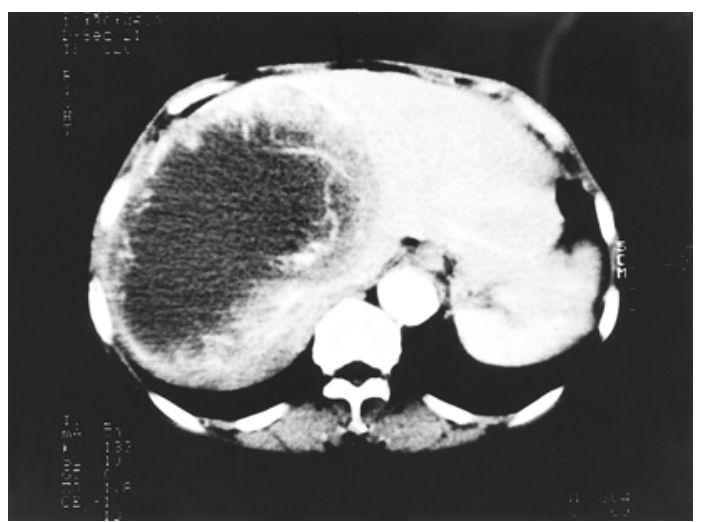

Figure 1. Intravenous bolus computed tomography of the abdomen showed a huge cystic mass with thick wall, occupying the most of the right lobe of the liver. The tumor wall has serpentine vascular enhancement during early contrast phase .

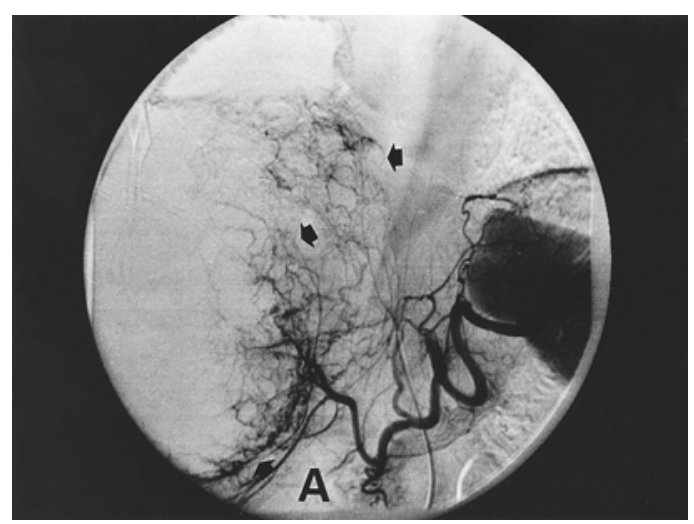

hepatomegaly with a huge space-occupying lesion in the right lobe. Neither splenic nor bone marrow uptake was increased. Technetium ${ }^{99} \mathrm{~m}$ RBC scan revealed no evidence of hepatic hemangioma.

Ultrasonography of the thyroid revealed a $1.5 \times 2.5 \times 3.5$ $\mathrm{cm}$ tumor in the right lobe, isodense with the gland parenchyma and having a $1.5 \mathrm{~cm}$ cystic lesion in the center. Color Doppler showed hyperdynamic blood flow around the lesion. Technetium ${ }^{99 \mathrm{~m}}$ pertechnetate thyroid scan showed a cold lesion in the right lobe. Small bowel series, barium enema and gastrofiberoscopy showed nothing unexpected.

Diagnostic needle aspiration of the liver mass produced clear amber-colored fluid with 50 white blood cells /HPF, all of which were lymphocytes; $30 \sim 40$ red blood cells /HPF; LDH $2135 \mathrm{IU} / \mathrm{L}$ and no visible malignant cell. Other biochemical findings of the fluid were nonspecific. Fine-needle aspiration biopsy and cytology of the thyroid mass showed follicular proliferation and cystic degeneration.

After laparotomy, a right hepatic lobectomy, wedge resection of the left lobe and cholecystectomy were done. The perihepatic, perigastric and omental veins were engorged, and hepatomegaly without cirrhotic change was noted. A huge mass bulged out from the right hepatic lobe and another mass, several centimeters in size, was found on segment 4. The portal veins and their branches appeared intact. No tumor was found on other organs. The patient was discharged well on the seventeenth postoperative day and remains free of recurrent hepatic tumor or other evidence of neoplasm 24 months after surgery.

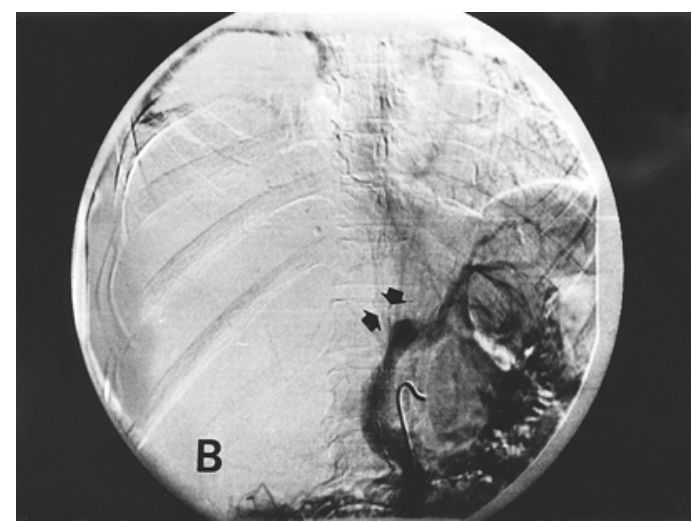

Figure 2. (A) Celiac angiography revealed a huge hypervascular mass in the right lobe of the liver. Tumor neovascularization was noted at the area of segments 4, 5, 6, 7 and 8. (B) Portovenography revealed total right portal vein defect. 
The resected specimen consisted of a whole right lobe of liver and a wedge-shaped portion of the left lobe. The external surface showed protruding bulging mass. On sectioning, a well-demarcated, grayish-white, multiloculated subcapsular tumor measuring $7.5 \times 16.0 \times 4.5 \mathrm{~cm}$ and having central cystic degeneration was noted. The surrounding solid portion was composed of soft, homogenous tissue with multifocal cystic and mucinous degeneration. The tumor showed an expanding growth pattern and the capsule was replaced by the expanding tumor mass. Several daughter nodules were noted (Figure 3).

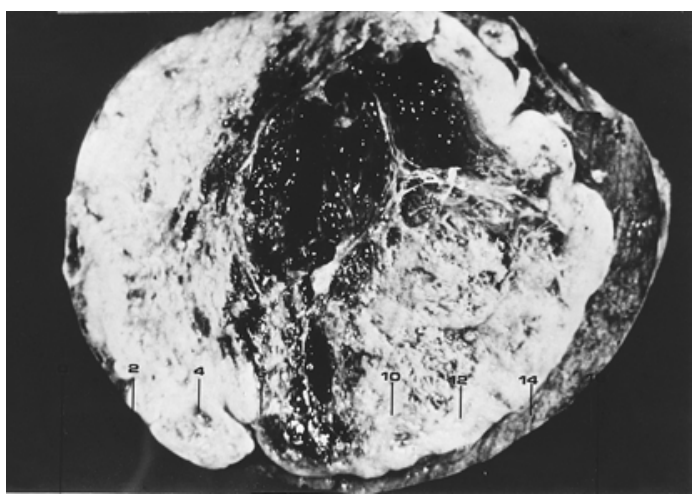

Figure 3. Gross examination of the excised tumor: Relatively well-demarcated, grayish-white mass measuring $7.5 \times 16.0 \times 4.5 \mathrm{~cm}$ in size showed expanding growth pattern. The tumor contained central cystic degeneration and homogeneous and homogenous solid portion.

Microscopic findings revealed anaplastic spindle cell proliferation with a diffuse myxochondroid background. The tumor cells had spindle-shaped, hyperchromatic, occasionally pleomorphic nuclei with frequent mitotic figures $(5 \sim 9 / H P F)$. Mild cellular pleomorphism with atypia was noted. Most areas consisted of tumor cells which had typical cigar-shaped nuclei with a prominent nucleolus and fibrillary cytoplasm arranged in interlacing bundles and short fascicles (Figure 4).

Occasionally, individual and/or aggregated primitive round tumor cells with vacuolated cytoplasm were scattered in the loose chondroid matrix (Figure 4). Small to mediumsized blood vessels were proliferated, showing a focal hemangiopericytic pattern. Immunohistochemical stains for vimentin, desmin, lysozyme and a1-antitrypsin were all positive, and keratin, actin and factor VIII were all
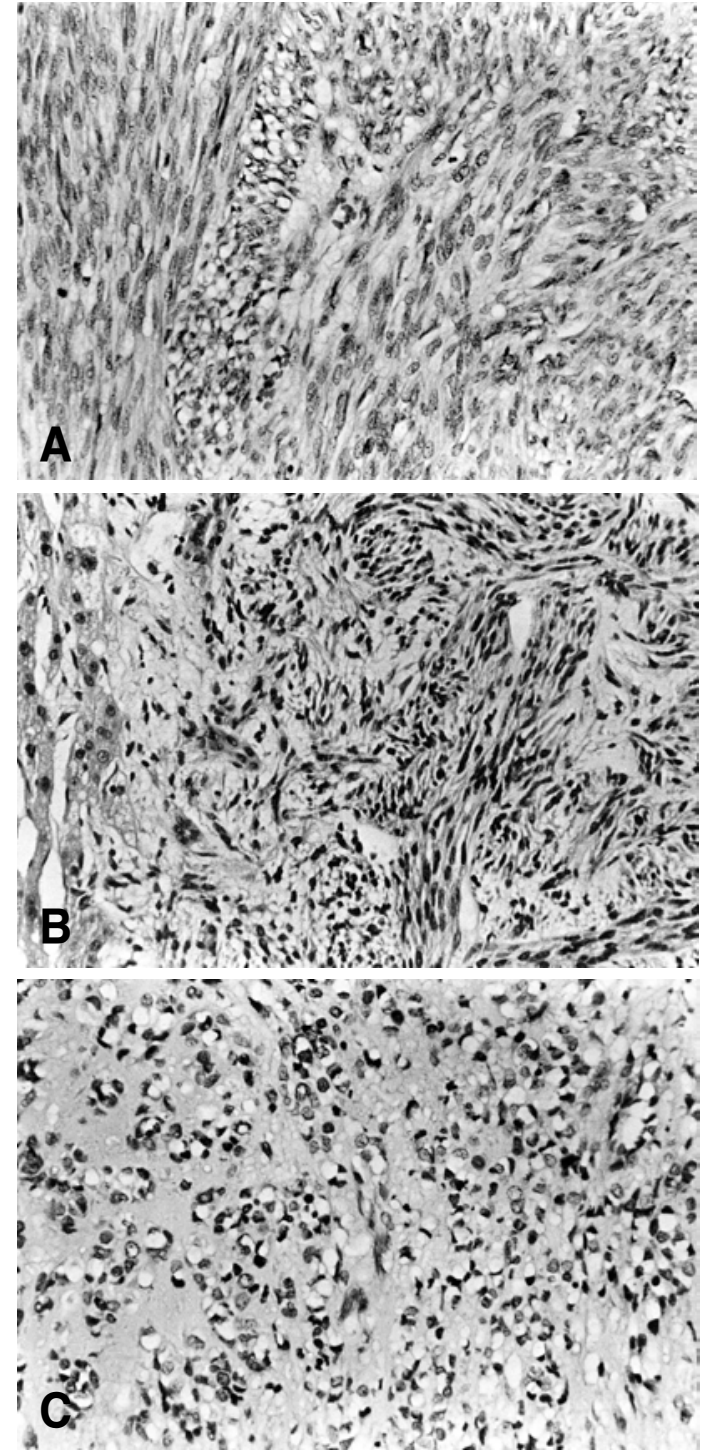

Figure 4. Light microscopic examination : (A) Sections of the liver mass showed interlacing fascicles of spindle-shaped nuclei and perinuclear vacuoles (H\&E, ×200) (B) Tumor showed interlacing spindle-shaped cells arranged in fascicles, with hepatocyte present in the left side. (H\&E, $\times 200$ ) (C) Tumor cells showed round nuclei with perinuclear vacuoles. The stroma revealed myxoid change. $(\mathrm{H} \& \mathrm{E}, \times 200)$

negative (Figure 5). Electronmicroscopic examination showed a well-developed basal lamina, plasma-membrane dense body, pinocytic vesicles and scattered dense filaments. These findings confirmed the myofilamentous nature of the tumor (Figure 6). 


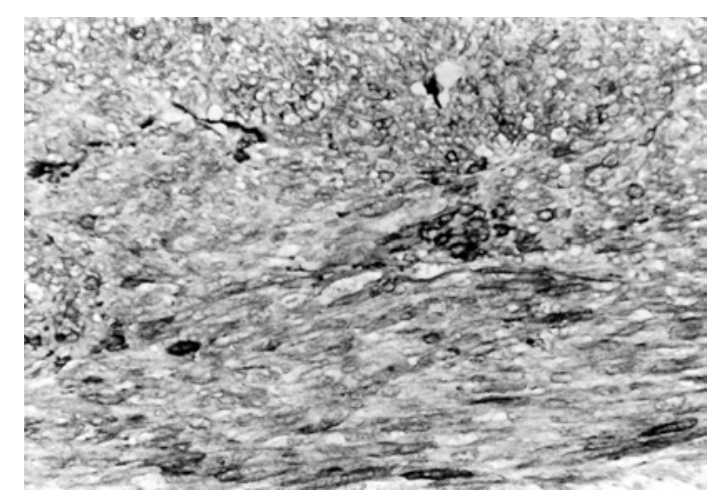

Figure 5. Immunohistochemical stains of the resected mass showed positive staining for smooth muscle actin. (Immunohistochemical stain, $\times 200$ )

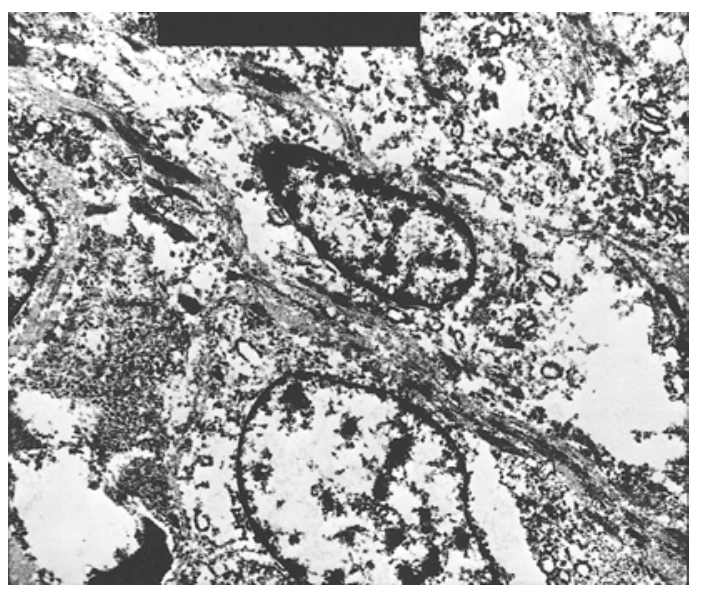

Figure 6. Electromicroscopic examination showed myofilaments (arrow) with dense bodies. (EM, ×6000)

\section{DISCUSSION}

Primary hepatic mesenchymal tumors are rare. Sarcomas occupy only $1 \%$ to $2 \%$ of all primary malignant tumors of the liver ${ }^{1,2)}$ and most of the primary hepatic malignant tumors are either hepatocellular carcinoma or cholangiocarcinoma. Nearly all primary sarcomas of the liver are angiosarcomas, epitheloid hemangioendotheliomas, undifferentiated embryonal sarcomas or leiomyosarcomas, with more than $70 \%$ being angiosarcoma or undifferentiated embryonal sarcoma ${ }^{2)}$.

Leiomyosarcomas are malignant neoplasms that arise from the smooth muscle. Focal or diffuse myxoid changes may occur in many types of benign and malignant soft tissue neoplasms. The myxoid variant of 12 leiomyosarcomas is distinctive and rare, with only 43 cases having been reported up to $1993^{5-7)}$. The frequent locations of the disease were the uterus (the most common; 28 cases), ovary (3 cases) and extragenital area (12 cases); i.e., digestive tract, retropertoneum, maxillary antrum, parotid gland and prostate ${ }^{5-7)}$.

Primary hepatic leiomyosarcoma is a very rare tumor occuring predominantly in elderly or middle-aged individuals ${ }^{14)}$, with fewer than 25 cases reported in the literature to date ${ }^{11,12)}$ and, to our knowledge, no primary myxoid leiomyosarcoma in the liver has been reported. Intrahepatic leiomyosarcoma may be derived from the smooth muscle cells of the bile ducts or blood vessels. No definite underlying etiologic factors are known, and the tumor is not associated with cirrhosis, although thorotrast associated lesions and an AIDS-related case in a young girl have been reported ${ }^{11,13)}$.

Usually, primary hepatic leiomyosarcoma is a large, solitary, no encapsulated mass. Massive tumor at the time of diagnosis has a size range of 6 to $35 \mathrm{~cm}$ and the typical weight is 1 to $5 \mathrm{~kg}$. The largest tumor reported was 11,270 g. The right lobe is the usual site ${ }^{14)}$. A large graysh white solitary mass with hemorrhage and necrosis is typical and a few satellite nodules may be noted. The Microscopic features of leiomyosarcoma are uniform spindle shaped or epithelioid smooth muscle cells with lightly eosinophilic cytoplasm, which display longitudinal striation. Nuclei are elongated or oval and exhibit hyperchromatism and pleomorphism with numerous mitoses: from one per 2 or $3 \mathrm{HPF}$ to 17 per $10 \mathrm{HPF}^{1,15)}$. Tumor cells are disposed in intersecting bundles. Positive cells on immunohistochemical stains for vimentin and desmin are negative for cytokeratin ${ }^{16-18)}$. In the myxoid variant, amorphous intercellular material that are positive on Alcian blue stain at $\mathrm{pH} 2.5$ and colloidal iron staining may represent stromal acid mucopolysaccharides ${ }^{3,6)}$. The myofilamentous nature of primary hepatic leiomyosarcomas can be confirmed by the ultrastructural features of well-developed basal lamina, plasma membrane dense bodies, micropinocytic vesicles, and dense filaments ${ }^{1,}{ }^{19}$ ).

To confirm the primary nature of leiomyosarcoma, it is necessary to exclude metastasis from extrahepatic site which is more common ${ }^{20)}$. Extrahepatic origin was not entirely excluded in the previous publications of liver leiomyosarcoma. But no extrahepatic tumor was identified in current case by abdominal and pelvic CT, small bowel contrast study, or laparotomy. In myxoid leiomyosarcoma, the sparse cellular density secondary to an abundant amount of extracellular substance makes a conventional 
mitotic count difficult and mitotic count is less useful as a marker of malignancy ${ }^{7}$.

Indeed, uterine myxoid leiomyosarcomas showing abnormally low mitotic counts have a great potential for aggression". For this reason, it has been suggested that mitotic count should be taken separately in the solid and myxoid areas ${ }^{6)}$ as, in the former, the counts are definitely higher. In the present case, the mitotic index in the solid area averaged 7 per $10 \mathrm{HPF}$, and the neoplasm was considered to be malignant according to the criterion of the mitotic index.

Space-occupying lesion (SOL) of the liver was detected in $1.24 \%$ of the general Korean population by screening ultrasonographic examination and $67.1 \%$ of SOLs were cystic lesions ${ }^{21}$. Cystic hepatic masses should be evaluated thoroughly to ensure accurate diagnosis and appropriate management for malignant tumors. Surgical resection is the most effective method of treatment but is usually not possible because of massive tumor. The majority of patients have recurrent massive hepatic tumor but the current case was well managed by effective surgical treatment.

\section{REFERENCES}

1) Maki HS, Hubert BC, Sajjad SM, Kirchner JP, Kuehner ME. Primary hepatic leiomyosarcoma. Arch Surg 122: 1193-1196, 1987

2) Haubrich WS, Schaffner F, Berk JE. Bokus Gastroenterology. 5th ed. Philadelphia: WB Saunders, 2488-2500, 1987

3) Watnuki K, Kusama K. [A case of leiomyosarcoma of the liver]. Nippon Ika Daigadu Zasshi 22:552, 1955

4) Tsuji M, Takenaka R, Kashihara T, Hadama T, Tere Wata N, Mori N. Primary hepatic leiomyosarcoma in a patient with hepatitis $C$ virus-related liver cirrhosis. Pathol Int 50(1):41-47, 2000

5) King ME, Dickersin GR, Schully RE. Myxoid leiomyosarcoma of the uterus. A report of 6 cases. Am J Surg Pathol 6:589-598, 1982

6) Salm R, Evans DJ. Myxoid leiomyosarcoma. Histopathology 9:159-169, 1985

7) Francisco FN, Alberto A, Isabel RA, Juan JS. Myxoid leiomyosarcoma of the ovary: analysis of three cases. Hum Pathol 22:1268-1273, 1991
8) Ilsoon K. Mortality patterns of major causes of death in Korea. J Korean Med Assoc. 38:132-145, 1995

9) Hyoseok L, Jikon R, Sookhyang J, Chungyang K. A prospective study on the incidence and the risk factors of the development of hepatocellular carcinoma in patients with liver cirrhosis in Korea. Korean J Gastroenterol 25:116-122, 1993

10) Changsik S, Ukyun N, Sangkweon O, Sungwon C, Chansup S. Diagnosis of simple hepatic cysts using ultrasonography. Korean J Gastroenterol 18:519-524, 1986

11) Ross JS, Del Rosario A, Bui HX, Sonbati H, Solis O. Primary hepatic leiomyosarcoma in a child with the acquired immunodeficiency syndrome. Hum Pathol 23:69-72, 1992

12) Paraskevopoulos JA, Stephenson TJ. Case report. Primary leiomyosarcoma of the liver. HPB Surg 4:157-163, 1991

13) Shurbaji MS, Olson JL, Kuhajda FP. Thorotrast-associated hepatic leiomyosarcoma and cholangiocarcinoma in a single patient. Hum Pathol 18:524-526, 1987

14) Fong J A, Ruebner B H. Primary leiomyosarcoma of the liver. Hum Pathol 5:115-119, 1974

15) Mclntyre N, Benthamou JP, Bircher J, Rizzetto M. Oxford Text Book of Clinical Hepatology, vol 2. Oxford: Oxford University Press, 1072-1073. 1991

16) Nakizoe S, Kojiro M, Nakashima T. Hepatocellular carcinoma with sarcomatous change: Clinicopathologic and immunohistochemical studies of 14 autopsy cases. Cancer 59:310-316, 1987

17) Johnson DE, Herndier BG, Medeiros LJ, Warnke RA, Rouse RV. The diagnostic utility of keratin profiles of hepatocellular carcinoma and cholangiocarcinoma. Am J Surg Pathol 12:187-197, 1988

18) Haratake J, Horie A. An immunohistochemical study of sarcomatoid liver carcinomas. Cancer 68:93-97, 1991

19) Bloustein PA. Hepatic leiomyosarcoma: Ultrastructural study and review of differential diagnosis. Hum Pathol 9:713-715, 1978

20) Hawkins FP, Jordan GL, McGavran MH. Primary leiomyoma of the liver: successful treatment by lobectomy and presentation of criteria for diagnosis. Am J Surg Pathol 4:301-304, 1980

21) Kim $K Y$, Lee $H J$, Suh J I, Back $C W$, Lee $D$ J, Song Y D, Doh G S, Jeon K J, Kim J H. A clinical study of liver space-occupying lesion among the visitors in an automated med-screening center by ultrasonographic examination. Kor J Med 51:178-186, 1996 